\title{
Preface of the special issue on the conference on computer-aided verification 2018
}

\author{
Hana Chockler ${ }^{1} \cdot$ Georg Weissenbacher ${ }^{2}$
}

Accepted: 15 February 2021 / Published online: 28 April 2021

(c) The Author(s), under exclusive licence to Springer Science+Business Media, LLC, part of Springer Nature 2021

This special issue of the Journal on Formal Methods in System Design features extended and revised versions of select contributions presented at the 2018 Conference on ComputerAided Verification [2,3]. CAV is the premier international conference on computer-aided verification, providing a forum for a broad range of advanced research in areas ranging model checking and automated theorem proving to testing, synthesis and related fields.

CAV 2018 was held from July 14 to July 17, 2018, in Oxford, UK. This special issue contains the following four contributions, all of which were reviewed by at least two experts in at least two rounds of peer review:

- Boolean Functional Synthesis: Hardness and Practical Algorithms, an extended version of [1], presents complexity results and novel algorithms to generate Skolem functions for Boolean functional synthesis.

- Model Checking Boot Code from AWS Data Centers, an extended version of [4], describes the authors' experience with symbolic model checking initial boot code running in data centers at Amazon Web Services.

- Quasi-Optimal Partial Order Reduction, an extended version of [5], explores the shortcomings of existing dynamic partial-order reduction approaches and presents a novel approach that can arbitrarily approximate an optimal exploration.

- On Solving Quantified Bit-Vector Constraints using Invertibility Conditions, an extended version of [6], presents a novel approach for solving quantified bit-vector constraints in Satisfiability Modulo Theories based on computing symbolic inverses of bit-vector operators.

Moreover, this issue also contains the announcement of the 2018 CAV Award, presented to Armin Biere, Alessandro Cimatti, Edmund M. Clarke, Daniel Kroening, Flavio Lerda, and Yunshan Zhu for outstanding contributions to the enhancement and scalability of model checking by introducing Bounded Model Checking based on Boolean Satisfiability for hardware and software.

\footnotetext{
Georg Weissenbacher

georg.weissenbacher@tuwien.ac.at

1 King's College London, London, UK

2 TU Wien, Vienna, Austria
} 
We thank the authors for their excellent work, all reviewers for their time and valuable suggestions for improving the manuscripts, and Daniel Kroening, the FMSD Editor in Chief, for his support.

\section{References}

1. Akshay S, Chakraborty S, Goel S, Kulal S, Shah S What's hard about Boolean functional synthesis? In: Chockler and Weissenbacher [2], pp 251-269. https://doi.org/10.1007/978-3-319-96145-3_14

2. Chockler H, Weissenbacher G (eds) (2018) Proceedings of the 30th international conference on computeraided verification (CAV) 2018, part I, lecture notes in computer science, vol 10981. Springer . https://doi. org/10.1007/978-3-319-96145-3

3. Chockler H, Weissenbacher G (eds) (2018) Proceedings of the 30th international conference on computeraided verification (CAV) 2018, part II, lecture notes in computer science, vol 10982. Springer. https://doi. org/10.1007/978-3-319-96142-2

4. Cook B, Khazem K, Kroening D, Tasiran S, Tautschnig M, Tuttle MR. Model checking boot code from AWS data centers. In: Chockler and Weissenbacher [3], pp 467-486. https://doi.org/10.1007/978-3-31996142-2_28

5. Nguyen HTT, Rodríguez C, Sousa M, Coti C, Petrucci L. Quasi-optimal partial order reduction. In: Chockler and Weissenbacher [3], pp 354-371. https://doi.org/10.1007/978-3-319-96142-2_22

6. Niemetz A, Preiner M, Reynolds A, Barrett CW, Tinelli C. Solving quantified bit-vectors using invertibility conditions. In: Chockler and Weissenbacher [3], pp 236-255. https://doi.org/10.1007/978-3-319-961422_16

Publisher's Note Springer Nature remains neutral with regard to jurisdictional claims in published maps and institutional affiliations. 